\title{
Engineering or Biology?
}

\author{
Miguel Burgos* \\ Department of Genetics, Faculty of Sciences, University of Granada, Spain
}

Received: June 01, 2015; Accepted: August 04, 2015; Published: September 08, 2015

*Corresponding author: Miguel Burgos, Department of Genetics, University of Granada, Labs 127 y A-105 CIBM, Biomedical Research Center, 18100 Armilla, Granada, Spain, Tel: +34-958-249-699; E-mail: mburgos@ugr.es

About ten thousand years ago, after the climate change at the end of the last glacial period, humans started producing their own food by farming instead of being nomadic hunters. This was the beginning of a long period in which artificial selection led to the domestication of many animal, vegetal, and even microbial species by indirectly manipulating their genotypes through phenotypic selection of traits of interest. Thus, we unconsciously started to genetically modify other species at the "neolithic revolution".

Over thousands of years, we did this job by selective breeding. For this artificial selection to work there must be an underlying genetic variation that was produced naturally and that required long periods of time to give results. When we knew about genes and genomes, DNA and mutations, we were able to randomly increase the occurrence of mutations (i.e. by irradiating seeds) and accelerate the process of finding new useful variants. At the early 50's we knew that the DNA is the genetic material [1]. One year later Watson and Crick [2] unravelled the structure of this molecule but we had to wait about 20 years more to be able to directly manipulate the DNA by means of techniques globally called as "Genetic Engineering" [3]. This first experiment managed to insert genetic information from the Simian virus 40 (SV40), a virus known for causing cancer, into Escherichia coli, a bacteria that is part of the human gut flora. The possibility of getting a cancer that spread due to bacterial infection alarmed the scientists who decided, at the Asilomar Conference in 1975 [4], that we should oversight further experiments until the recombinant DNA technology was safe. During this voluntary moratorium, the National Institutes of Health in USA developed the guidelines for this technology, which was then considered safe enough. In 90's, Genetic Engineering and its vocabulary was common on daily press and television.

This technology has lead to great advances in biomedical research, agriculture and industry among others, although some of them are not free of controversy. Some examples of proteins with therapeutical applications produced by recombinant DNA technology are Human Insulin, Growth Hormone, Interferons, Erythropoietin, Blood Clotting Factor VIII, Platelet Growth Factor, etc., that are now obtained from Genetically Modified Organisms (GMO). Antigens for the development of vaccines such as the hepatitis B surface antigen, Cholera toxin B subunit, Influenza virus haemagglutinin, Rabies glycoprotein, etc. are also outcomes of this technology. On the agricultural side, it has produced rice engineered to synthesize $\beta$-carotene, crops resistant to the herbicide glyphosate or crops producing Bt-toxin for insect-control.

Despite the common use of restriction enzymes, ligase and cloning vectors, Genetic engineering lacked the standardization of other engineering disciplines. In the early 2000's, a new philosophy emerged aiming at applying the principles of engineering: abstraction, standardization and quality control, to the Genetic Engineering. Tom Knight, from the MIT Computer Science and Artificial Intelligence Laboratory, published a report entitled "Idempotent Vector Design for standard Assembly of BioBricks" [5]. The first paragraph of his report stated that:

The lack of standardization in assembly techniques for DNA sequences forces each DNA assembly reaction to be both an experimental tool for addressing the current research topic, and an experiment in and of itself. One of our goals is to replace this ad hoc experimental design with a set of standard and reliable engineering mechanisms to remove much of the tedium and surprise during assembly of genetic components into larger systems.

Standard biological parts called "BioBricks" are pieces of DNA that form a functional unit such as promoters, coding sequences, terminators etc. They can be combined with vectors that allow assembling these parts in the desired order applying consecutively the same standardized techniques. The combination of different parts makes a "device" and several devices a "system". This standardization gave birth to the "Synthetic Biology", a discipline that can be defined as the design and construction of new biological parts, devices, and systems, and the re-design of existing, natural biological systems for useful purposes [6]. To promote the use and knowledge about Synthetic Biology among undergraduate students the MIT group developed the "International Genetically Engineered Machines" (iGEM) competition [7] and pioneered "The Registry of Standard Biological Parts" [8], a collection of a few thousand BioBrick parts. Most parts of the registry are contributions of the iGEM teams that attend the competition and are shared with the community. Hundreds of projects have been contributed by iGEM teams revealing the incredible potential of the Synthetic Biology. For example, the Edinburg team designed a biosensor that detects different concentrations of arsenic and 
emit a response as a pH signal for the 2006 iGEM competition [9]. The Berkeley's team developed bacterial containers of hemoglobin that work as artificial blood in 2007 [10]. The Valencia team presented in 2009 a display made of engineered yeasts that responded to electrical signals emitting luminescence and that was able to display simple images in slow movement [11]. Also, this same year the Cambridge team presented a E. coli genetically engineered to produce a variety of colored pigments that they called Escherichia chrome [12]. The Sevilla team project for the 2011 competition was the design of bacteria that can operate as logic gates and thus per-form simple logical operations [13]. In 2013, the Freiburg team developed a toolkit that enables customizable gene regulation in mammalian cells based on the CRISPR/Cas9 system [14].

The standardization of reusable parts and methods allows students to develop complex projects in few months. The potential of the Synthetic Biology is enormous, and can engineer from scratch complex genetic circuits, metabolic pathways or even whole genomes. A search in the Web of Science for papers mentioning Synthetic Biology reveals that from the few papers that appeared in 2004, the number of publications have exponentially grown to near 900 in 2014 and the number of citations to more than 11000 .

In the words of Tom Knight: This is going to change how we build things. Biology is fundamentally a manufacturing technology, and we are on the verge of figuring out how to control that. It's impossible to predict and estimate the impact of that, but it's going to be massive.

\section{References}

1. Hershey $A D$, Chase $M$. Independent functions of viral protein and nucleic acid in growth of bacteriophage. J Gen Physiol. 1952; 36(1):39-56.

2. Watson JD, Crick FH. Molecular structure of nucleic acids; a structure for deoxyribose nucleic acid. Nature. 1953; 171(4356):737-8.

3. Jackson DA, Symons RH, Berg P. Biochemical method for inserting new genetic information into DNA of Simian Virus 40: circular SV40 DNA molecules containing lambda phage genes and the galactose operon of Escherichia coli. Proc Natl Acad Sci U S A. 1972; 69(10):2904-9.

4. Berg P, Baltimore D, Brenner S, Roblin RO, Singer MF. Summary statement of the Asilomar Conference on recombinant DNA molecules. Proc Natl Acad Sci U S A. 1975; 72(6):1981-4.

5. http://hdl.handle.net/1721.1/21168.

6. http://syntheticbiology.org/.

7. http://igem.org/

8. http://partsregistry.org/.

9. http://2006.igem.org/wiki/index.php/Arsenic_Biosensor.

10.http://2007.igem.org/Berkeley_UC.

11.http://2009.igem.org/Team:Valencia/home.

12.http://2009.igem.org/Team:Cambridge/Project/Pigments. 13. http://2011.igem.org/Team:Sevilla/Project.

14.http://2013.igem.org/Team:Freiburg. 